\title{
JunB expression is a common feature of CD30 + lymphomas and lymphomatoid papulosis
}

\author{
George Z Rassidakis, Athanasios Thomaides, Coralyn Atwell, Richard Ford, Dan Jones, \\ Francois-Xavier Claret and L Jeffrey Medeiros
}

Department of Hematopathology, The University of Texas MD Anderson Cancer Center, Houston, TX, USA

\begin{abstract}
JunB is a member of the Jun family of proteins that are components of the AP-1 transcription factor complex. AP-1 is involved in cell proliferation and apoptosis. Recent evidence suggests that Hodgkin and ReedSternberg cells overexpress JunB and that JunB facilitates constitutive CD30 expression by binding to an AP-1 site in the $C D 30$ promoter. In this study we surveyed JunB expression in a variety of CD30 + lymphoma types including 42 cases of anaplastic large cell lymphoma, 36 classical Hodgkin lymphoma, 15 cutaneous anaplastic large cell lymphoma, and 11 CD30 + diffuse large B-cell lymphoma. In addition, seven cases of nodular lymphocyte-predominant Hodgkin lymphoma and 42 diffuse large B-cell lymphoma, known to be CD30-, were analyzed. JunB expression was assessed using tissue microarrays, immunohistochemistry and a monoclonal antibody specific for JunB. Expression of JunB was observed in 41 of 42 cases of anaplastic large cell lymphoma, including all 21 cases positive for anaplastic lymphoma kinase and 20 of 21 (95\%) negative for anaplastic lymphoma kinase. JunB was also expressed in all cases of classical Hodgkin lymphoma, cutaneous anaplastic large cell lymphoma and CD30 + diffuse large B-cell lymphoma, and in lymphomatoid papulosis. By contrast, all nodular lymphocyte-predominant Hodgkin lymphomas and diffuse large B-cell lymphomas that were CD30 - were also JunB -. We conclude that JunB is expressed in virtually all CD30 + lymphomas and is a potential target for experimental therapy in patients with these tumors.

Modern Pathology (2005) 18, 1365-1370. doi:10.1038/modpathol.3800419; published online 6 May 2005
\end{abstract}

Keywords: JunB; CD30; anaplastic large cell lymphoma; Hodgkin lymphoma; lymphomatoid papulosis

Activator protein $1(\mathrm{AP}-1)$ is a transcription factor complex composed of a group of proteins that bind to AP-1 DNA recognition elements, thereby inducing expression of genes controlling cell growth and apoptosis. ${ }^{1}$ AP-1 proteins include four subfamilies, Jun, Fos, Maf and ATF, and these proteins function as a complex of homodimers and heterodimers. ${ }^{1}$ The Jun family includes three members, c-Jun, JunB and JunD. The JunB gene is mapped at chromosome $19 \mathrm{p} 13$ and encodes a $39 \mathrm{kDa}$ protein. The main difference between JunB and c-Jun proteins is in their DNA binding domains where a small number of amino acid changes leads to a 10-fold decrease in the DNA binding ability of JunB. As a result, JunB is less stable and therefore less effective as a transcrip-

Correspondence: Dr LJ Medeiros, MD, Department of Hematopathology, Box 72, The University of Texas MD Anderson Cancer Center, 1515 Holcombe Blvd, Houston, TX 77030, USA.

E-mail: jmedeiro@mail.mdanderson.org

Financial support: Dr GZ Rassidakis is a recipient of an Odyssey Program Special Fellowship provided by The University of Texas MD Anderson Cancer Center.

Received 4 January 2005; revised 25 February 2005; accepted 3 March 2005; published online 6 May 2005 tion activator. Since JunB represses the transactivation and transformation capacity of c-Jun by forming inactive heterodimers with c-Jun, it has been suggested that cJun-JunB complexes may suppress cell proliferation. ${ }^{2,3}$ However, in specific cell types JunB can also act as a positive cell growth regulator in the absence of c-Jun. ${ }^{1}$

Little is known about the role of JunB in the pathogenesis of lymphomas. Mathas et $a l^{4}$ have shown that AP-1 is constitutively activated in Hodgkin and Reed-Sternberg cells of Hodgkin lymphoma with overexpression of functional c-Jun and JunB. These authors suggested that c-Jun and JunB expression may be a 'hallmark' of Hodgkin and Reed-Sternberg cells. ${ }^{4}$ In a more recent in vitro study, JunB was found to interact with an AP-1 site in the microsatellite sequence of the $C D 30$ gene promoter thereby facilitating $C D 30$ expression in Hodgkin and Reed-Sternberg cells. ${ }^{5}$ Among nonHodgkin lymphomas, a subset of cases of cutaneous lymphoma including cutaneous anaplastic large cell lymphoma were reported to overexpress JunB. ${ }^{6}$

In this study, we surveyed JunB expression in a variety of $\mathrm{CD} 30+$ lymphoma types and in 
lymphomatoid papulosis. We have shown that JunB overexpression is a common finding in virtually all CD30 + lymphomas. These results suggest that JunB is necessary for CD30 expression and that JunB has a potential pathogenetic role in these neoplasms.

\section{Materials and methods}

\section{Lymphoma Cell Lines and Tumor Specimens}

A panel of CD30 + lymphoma cell lines were used including three anaplastic lymphoma kinase (ALK) + anaplastic large cell lymphoma (Karpas 299, JB-6 and SU-DHL-1), one ALK- anaplastic large cell lymphoma (Mac2A), and five Hodgkin lymphoma, (L428, L1236, KM-H2, HD-MyZ, and HD-LM2). In addition, Jurkat, a T-cell acute lymphoblastic leukemia cell line known to be CD30-, was used as a negative control. All cell lines were grown in suppplemented Roswell Park Memorial Institute 1640 medium (Life Technologies, Grand Island, NY, USA) as described previously.

Western blot analysis and flow cytometry (for CD30) were performed on these cell lines as described elsewhere. ${ }^{7,8}$ For Western blotting, the JunB monoclonal antibody (C-11, Santa Cruz Technology, Santa Cruz, CA, USA) at a 1:200 dilution and the anti-CD30 monoclonal antibody (Ber-H2, Dakocytomation, Carpinteria, CA, USA) at a 1:25 dilution were used. For flow cytometry, a monoclonal CD30 antibody conjugated to phycoerythrin (Immunotech, Westbrook, ME, USA) was used non-diluted. Expression of JunB was judged to be negative (-), faint $(+/-)$, moderate $(+)$, and strong $(++)$ in immunoblots.

The tumors assessed included 153 cases of lymphoma and 18 cases of lymphomatoid papulosis accessioned at The University of Texas MD Anderson Cancer Center. The CD30 + group included 42 anaplastic large cell lymphomas (21 ALK +, 21 ALK-), 36 classical Hodgkin lymphomas, 15 cutaneous anaplastic large cell lymphomas, 11 CD30 + diffuse large B-cell lymphomas, and 18 cases of CD30 + lymphomatoid papulosis. For comparison, 49 CD30- lymphomas were assessed including seven nodular lymphocyte predominant Hodgkin lymphomas and 42 diffuse large B-cell lymphomas. ALK expression was assessed in all 42 anaplastic large cell lymphomas using the ALK-1 antibody (Dakocytomation). The diagnosis of all lymphomas was based on morphologic and immunohistologic criteria as specified in the World Health Organization classification. ${ }^{9}$

\section{Tissue Microarrays and Immunohistochemical Methods}

Cell pellets from the lymphoma cell lines were fixed in $4 \%$ buffered formalin overnight and embedded in paraffin. These cell blocks were then used to construct a 'cell line' microarray.
The tissue microarrays included duplicate, triplicate or quadruplet tumor cores from all cases of lymphoma and lymphomatoid papulosis. Two reactive lymph nodes were also assessed. A manual tissue arrayer (Beecher Instruments, Silver Springs, MD, USA) was used to construct these cell line and tissue microarrays as described previously. ${ }^{10}$ The immunohistochemical methods used in this study have been described previously. ${ }^{11}$ Heat-induced epitope retrieval was performed. Slides were incubated with a JunB monoclonal antibody (C-11, Santa Cruz Biotechnology) at a 1:100 dilution overnight at $4^{\circ} \mathrm{C}$. Detection of the immunoreaction was performed using the LSAB + kit (Dakocytomation) and $3,3^{\prime}$ diaminobenzidine $/ \mathrm{H}_{2} \mathrm{O}_{2}$.

In a subset of five classical Hodgkin lymphoma and 18 ALK + anaplastic large cell lymphoma cases, double immunostaining for JunB and CD30 was performed using the peroxidase/alkaline phosphatase-based EnVision Doublestain system (Dakocytomation) according to the manufacturer's recommended protocol. The slides were initially incubated with JunB antibody and then with the CD30 antibody at room temperature, each for 30 min. $3,3^{\prime}$ diaminobenzidine $/ \mathrm{H}_{2} \mathrm{O}_{2}$ and fast red were used as chromogens, respectively.

\section{Results}

\section{JunB Protein Expression in CD30 + Lymphoma Cell Lines}

Western blot analysis demonstrated that all anaplastic large cell lymphoma and Hodgkin lymphoma cell lines expressed JunB protein (Figure 1a, Table 1). One anaplastic large cell lymphoma (JB-6) and two Hodgkin lymphoma (L-1236 and HD-MyZ) expressed relatively lower levels of JunB compared

Table 1 JunB expression in CD30+ lymphoma cell lines

\begin{tabular}{llccc}
\hline Cell line & \multirow{2}{*}{$\begin{array}{l}\text { Lymphoma } \\
\text { type }\end{array}$} & $\begin{array}{c}\text { CD30 expression } \\
(F C \text { and } W B)\end{array}$ & Jun B expression \\
\cline { 4 - 5 } & & & $W B$ & IHC \\
\cline { 4 - 5 } & & + & ++ & ++ \\
Karpas 299 & ALK+ALCL & + & ++ & ++ \\
SU-DHL1 & ALK+ALCL & + & + & ND \\
JB-6 & ALK+ALCL & + & ++ & ++ \\
Mac2A & ALK-ALCL & + & ++ & ++ \\
L-428 & HL & + & + & + \\
L-1236 & HL & + & + & ND \\
KM-H2 & HL & + & + & ND \\
HD-MyZ & HL & + & - & - \\
HD-LM2 & HL (T-cell) & $+/-$ & - & +- \\
Jurkat & T-ALL & - & &
\end{tabular}

FC, flow cytometry; WB, Western blot analysis; ALK, anaplastic lymphoma kinase; ALCL, Anaplastic large cell lymphoma; HL, Hodgkin lymphoma; T-ALL, T-acute lymphoblastic leukemia; ND, not done.

IHC, Immunohistochemistry performed on paraffin-embedded cell blocks as described in the Methods section. All JunB-positive cell lines assessed showed strong nuclear immunoreactivity for JunB except L-1236 which was weakly positive. 


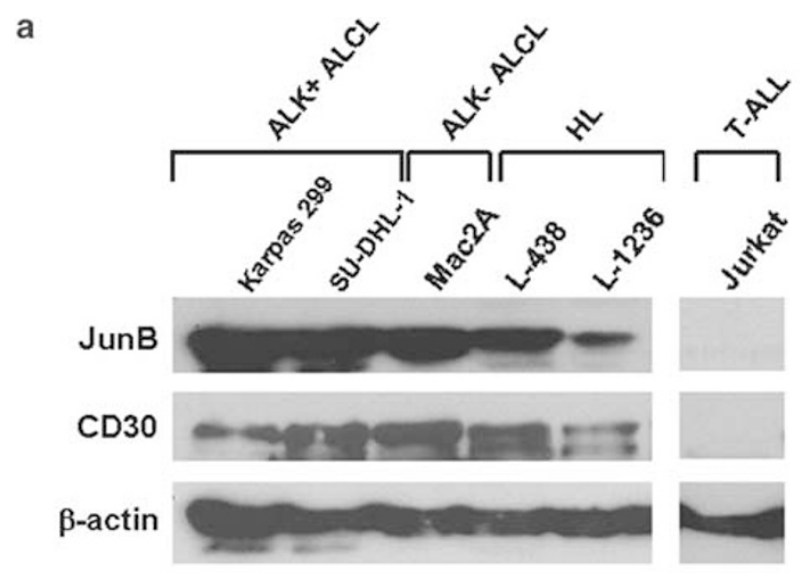

b

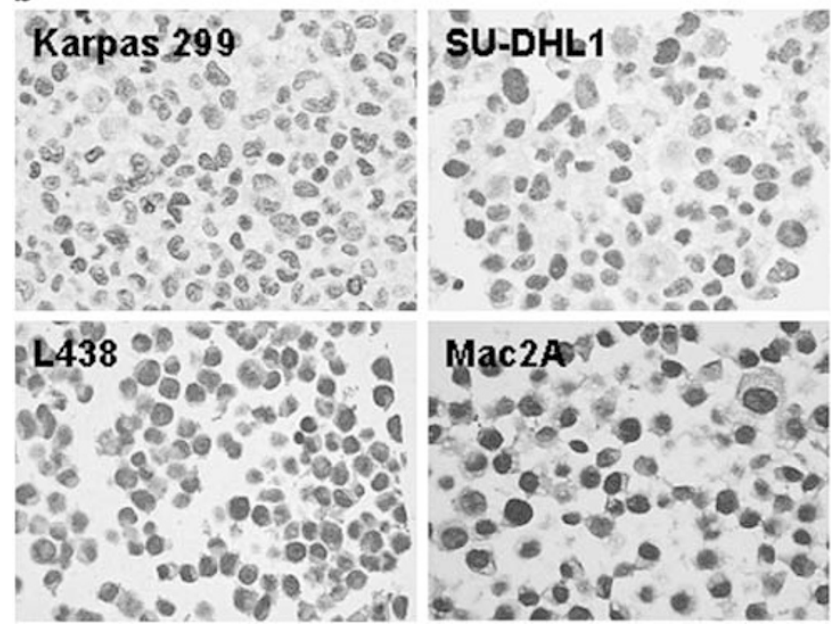

Figure 1 Expression of JunB in CD30 + lymphoma cell lines. (a) Immunoblots showing the level of expression of JunB protein in CD30 + cell lines. Jurkat cells (known to be CD30-) were JunB-. The same PVDF membrane was probed with a CD30 monoclonal antibody. $\beta$-actin was used as a control for protein load and integrity. (b) JunB expression was also detected by immunohistochemistry performed on sections cut from a lymphoma cell line array. JunB immunostaining perfectly correlated with the Western blot data. Representative examples of CD30 + cell lines including anaplastic large cell lymphoma and Hodgkin lymphoma are shown (immunoperoxidase, DAB).

with the other anaplastic large cell lymphoma cell lines. HD-LM2, a Hodgkin lymphoma cell line of T-cell origin, expressed JunB (and CD30) faintly (Table 1). Jurkat cells, known to be CD30-, were JunB-. To further confirm these results, we analyzed a cell line microarray that included these cell lines using immunohistochemistry and the same monoclonal antibody as was used for Western blot analysis. The immunostaining results correlated well with the Western blot results (Figure 1b, Table 1).

\section{Expression of JunB in Reactive Lymph Nodes, CD30 + Lymphomas and Lymphomatoid Papulosis}

In reactive lymphoid follicles, JunB was faintly expressed in rare germinal center cells and small lymphocytes (Figure 2a, inset). The staining pattern was exclusively nuclear.
All 21 ALK + anaplastic large cell lymphomas and 20 of 21 (95\%) ALK-anaplastic large cell lymphomas strongly and uniformly expressed JunB (Figure 2b and c, Table 2). Similarly, all 36 Hodgkin lymphomas, 15 cutaneous anaplastic large cell lymphomas, 11 CD30 + diffuse large B-cell lymphomas, and 18 cases of lymphomatoid papulosis strongly and uniformly expressed JunB in the nucleus of tumor cells (Table 2). By contrast, all 49 CD30- tumors, seven nodular lymphocyte-predominant Hodgkin lymphomas and 42 diffuse large B-cell lymphomas, were JunB(Figure 2d and f, Table 2).

In a subset of five classical Hodgkin lymphoma and $18 \mathrm{ALK}+$ anaplastic large cell lymphoma cases, double immunostaining for JunB and CD30 was performed. Coexpression of JunB (nuclear) and CD30 (membranous and cytoplasmic) was seen in the Hodgkin and Reed-Sternberg cells of classical Hodgkin lymphoma and the tumor cells of ALK+ anaplastic large cell lymphoma in all cases (Figure 3a-d). Rare CD30- small reactive lymphocytes showed faint immunoreactivity for JunB (Figure 3).

\section{Discussion}

The AP-1 transcription factor complex is a group of proteins capable of forming homodimers or heterodimers through their leucine-zipper domains. Different combinations of homodimers and heterodimers recognize different sequences in the promoters of AP-1 target genes that control cell proliferation and apoptosis thus contributing to oncogenesis. ${ }^{12}$ Of the proteins involved in AP-1, the Jun family, and particularly JunB, is the focus of this study.

Recent studies suggest a potential role for the AP-1 family of proteins, and in particular, the Jun family in the pathogenesis of Hodgkin lymphoma. ${ }^{4}$ In cultured Hodgkin and Reed-Sternberg cells, JunB was found to bind with the $C D 30$ gene promoter. The CD30 gene promoter has three regions: a microsatellite sequence containing CCAT repeats, a core promoter with Sp-1 binding sites, and a downstream promoter element. In normal lymphoid cells, the microsatellite sequence suppresses the CD30 promoter. However, in Hodgkin and ReedSternberg cells JunB binds to an AP-1 site in the microsatellite sequence resulting in relief of repression of the $C D 30$ promoter. $^{5}$ As a result, $C D 30$ gene expression is induced in Hodgkin and Reed-Sternberg cells.

Based on these data, we hypothesized that JunB might be overexpressed in other CD30 + lymphoproliferative disorders. We therefore assessed for JunB expression in a variety of CD30 + lymphomas including Hodgkin lymphoma and different types of CD30 + non-Hodgkin lymphoma. We also assessed cases of lymphomatoid papulosis. We found that 41 of $42(98 \%)$ anaplastic large cell lymphoma cases were JunB + (Table 1). In addition, all cases of 

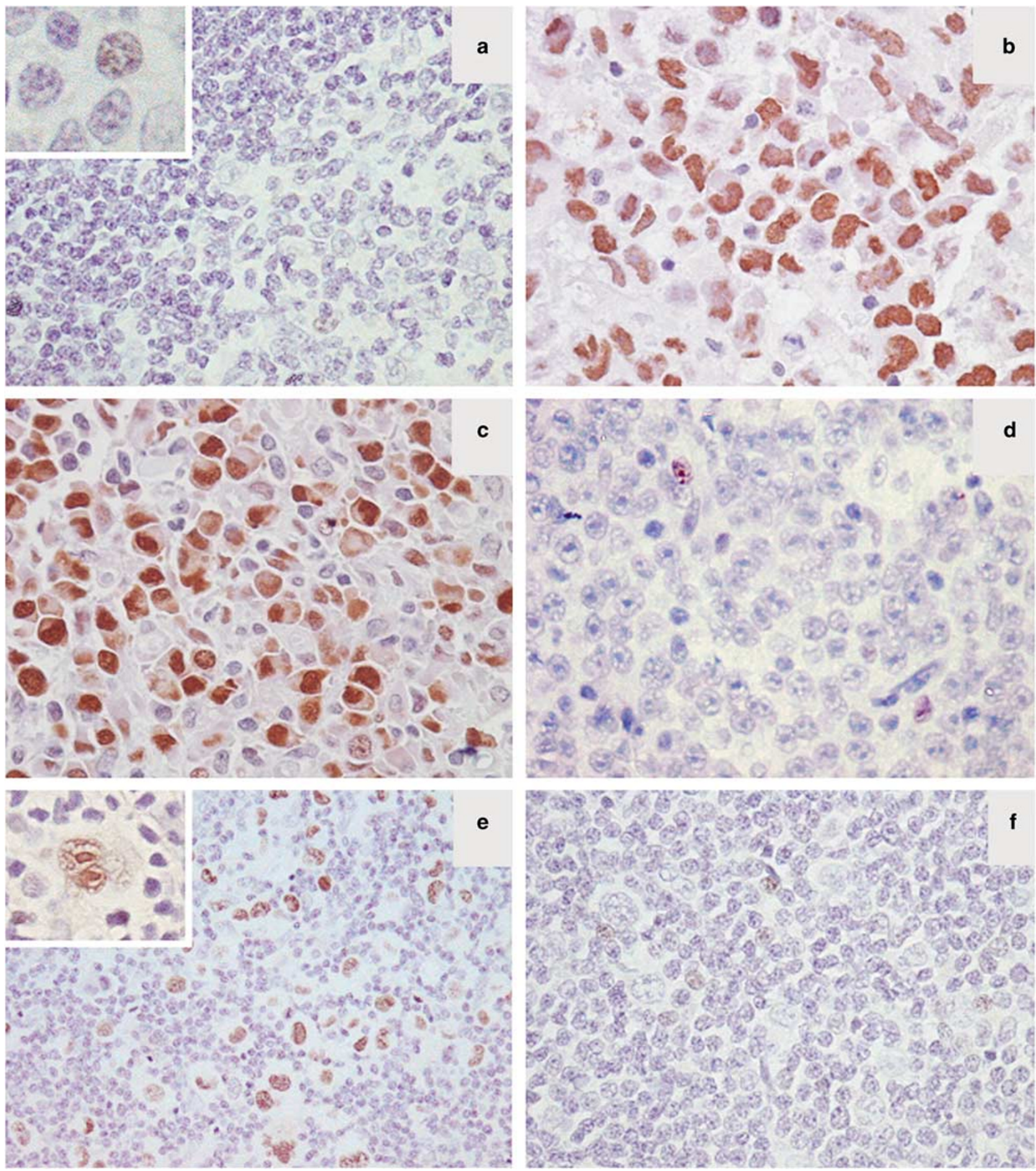

Figure 2 Expression of JunB in reactive lymph nodes and CD30 + lymphomas. (a) Reactive lymphoid follicles are mostly negative for JunB. Faint nuclear JunB expression is seen only in occasional reactive lymphocytes (inset). (b,c) Representative cases of ALK + (b) and an ALK - (c) anaplastic large cell lymphoma strongly positive for JunB. (d) A CD30- diffuse large B-cell lymphoma that did not express JunB is shown. (e) A case of nodular sclerosis classical Hodgkin lymphoma with strong expression of JunB in the neoplastic Hodgkin and Reed-Sternberg cells. (f) JunB was not expressed in the tumor cells of lymphocyte predominant Hodgkin lymphoma as shown. A few reactive lymphocytes are weakly positive in $\mathbf{d}$ and $\mathbf{f}$.

classical Hodgkin lymphoma, CD30 + diffuse large B-cell lymphoma, cutaneous anaplastic large cell lymphoma and lymphomatoid papulosis expressed JunB. Furthermore, we demonstrated coexpression of JunB and CD30 by the Hodgkin and ReedSternberg cells of classical Hodgkin lymphoma and the tumor cells of ALK + anaplastic large cell lymphoma using a double immunostaining 
Table 2 JunB expression in CD30+ lymphomas and lymphomatoid papulosis

\begin{tabular}{|c|c|c|c|}
\hline Lymphoma type & Number of tumors & $C D 30+(\%)$ & $J u n B+(\%)$ \\
\hline Anaplastic large cell lymphoma & 42 & & \\
\hline ALK+ + & 21 & $21(100)$ & $21(100)$ \\
\hline ALK- & 21 & $21(100)^{1}$ & $20(95)$ \\
\hline Primary cutaneous anaplastic large cell lymphoma & 15 & 15 & $15(100)$ \\
\hline Hodgkin lymphoma & 43 & & \\
\hline Classical Hodgkin lymphoma & 36 & $36(100)$ & $36(100)$ \\
\hline Nodular lymphocyte predominant Hodgkin lymphoma & 7 & $0(0)$ & $0(0)$ \\
\hline CD30+ diffuse large B-cell lymphoma & 11 & $11(100)$ & $11(100)$ \\
\hline Mediastinal diffuse large B-cell lymphoma & 1 & 1 & 1 \\
\hline Diffuse large B-cell lymphoma with anaplastic features & 2 & 2 & 2 \\
\hline CD30+ diffuse large B-cell lymphoma post-transplant & 1 & 1 & 1 \\
\hline Other CD30+ diffuse large B-cell lymphomas & 7 & 7 & 7 \\
\hline CD30- diffuse large B-cell lymphoma & 42 & $0(0)$ & $0(0)$ \\
\hline Lymphomatoid papulosis & 18 & $18(100)$ & $18(100)$ \\
\hline
\end{tabular}
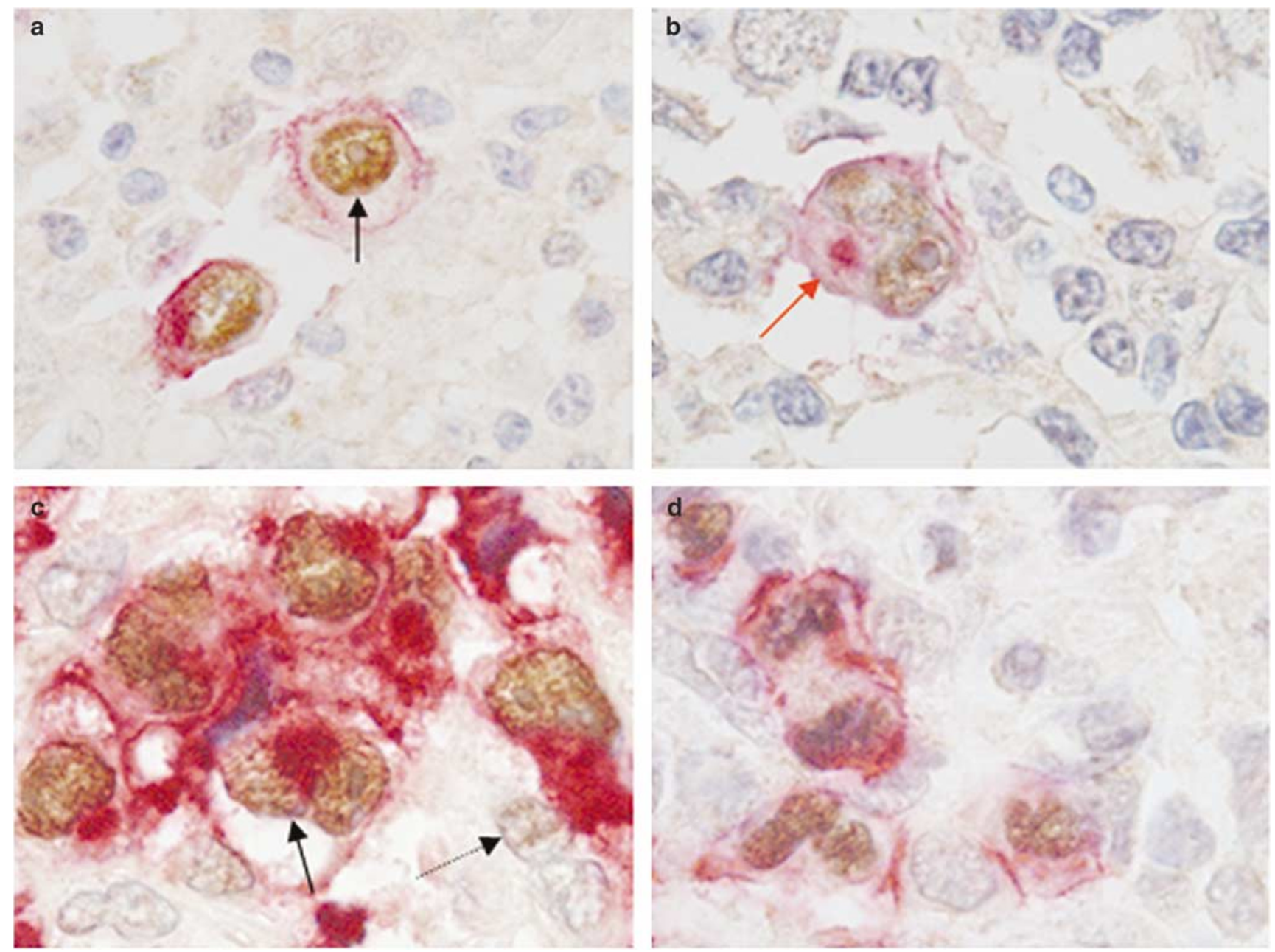

Figure 3 Coexpression of JunB and CD30 in classical Hodgkin lymphoma and ALK + anaplastic large cell lymphoma. (a,b) Double immunostaining showing nuclear expression JunB (dark brown, black arrow) and membranous/cytoplasmic (Golgi) expression of CD30 (red, red arrow) in Hodgkin and Reed-Sternberg cells of classical Hodgkin lymphoma. (c,d) Representative examples of ALK+ anaplastic large cell lymphoma tumors that are strongly positive for nuclear JunB (dark brown, black arrow) and CD30 (red) expression. Faint nuclear JunB expression is also seen in occasional reactive lymphocytes (light brown, dotted arrow). 
technique. By contrast, all CD30- cases of nodular lymphocyte-predominant Hodgkin lymphoma and diffuse large B-cell lymphoma were JunB- (Table 1). In all tumors assessed, we also identified rare CD30 - small reactive lymphocytes that showed faint nuclear immunoreactivity for JunB indicating very low expression levels. The biologic significance of this finding is uncertain.

The results of this survey are in agreement with previously published data. Mathas et $a l^{4}$ have shown overexpression of JunB in Hodgkin lymphoma. Moreover, a subset of cutaneous T-cell lymphomas including cutaneous anaplastic large cell lymphoma tumors also express JunB.$^{6}$ It is tempting to speculate that JunB, in CD30 + non-Hodgkin lymphomas and lymphomatoid papulosis, induces CD30 expression through interacting with the CD30 gene promoter, as has been shown in Hodgkin and Reed-Sternberg cells of Hodgkin lymphoma. ${ }^{5}$

The underlying mechanism(s) of JunB overexpression in CD30 + lymphomas and lymphomatoid papulosis are largely unknown. Mao et $a l^{6}$ used comparative genomic hybridization to show gain of chromosomal material at the 19p13 locus, the site of the JunB gene. They also used real-time PCR to show JunB overexpression. Thus, gene amplification is one possibility. However, JunB gene amplification has not yet been reported in Hodgkin lymphoma or other $\mathrm{CD} 30+$ non-Hodgkin lymphomas. Another theoretical mechanism would be accumulation of JunB protein due to decreased JunB degradation in these tumors. Recent studies provide insight into the mechanisms of c-Jun and JunB turnover. ${ }^{13,14}$ Interestingly, Gao et $a l^{14}$ demonstrated that JunB (and c-Jun) abundance can be regulated by extracellular stimuli in T-cells through Jun-N terminal kinase (JNK)-dependent phosphorylation of the novel E3 ligase Itch.

The role of JunB-related AP-1 dimers in controlling cell proliferation is not completely understood. Although it has been suggested that JunB can form complexes with c-Jun that could suppress cell proliferation, ${ }^{2,15}$ the effects of JunB and its complexes with other AP-1 members on cell growth are still under investigation. ${ }^{1}$ Recent evidence in chronic myeloid leukemia suggests that JunB activity is significantly decreased as compared with the peripheral blood cells of healthy individuals, and that downregulation of JunB is the result of JunB gene methylation. ${ }^{16}$ Furthermore, in mice JunB inactivation results in a myeloproliferative disorder resembling chronic myeloid leukemia. ${ }^{17}$ Szremska et $a l^{18}$ also have reported that transgenic expression of JunB inhibits proliferation and transformation in B but not $\mathrm{T}$ lymphocytes suggesting differential effects of JunB among different lymphocyte subtypes.

In summary, we have shown that JunB overexpression is a virtually constant feature in CD30 + lymphomas and in lymphomatoid papulosis. More mechanistic studies are needed to shed light on the molecular mechanisms underlying JunB upregula- tion as well the potential oncogenic role of JunB/ CD30 signaling in these lymphomas.

\section{References}

1 Shaulian E, Karin M. AP-1 as a regulator of cell life and death. Nat Cell Biol 2002;4:E131-E136.

2 Schutte J, Viallet J, Nau M, et al. jun-B inhibits and Cfos stimulates the transforming and trans-activating activities of c-jun. Cell 1989;59:987-997.

3 Deng T, Karin M. JunB differs from c-Jun in its DNAbinding and dimerization domains, and represses $\mathrm{C}^{-}$ Jun by formation of inactive heterodimers. Genes Dev 1993;7:479-490.

4 Mathas S, Hinz M, Anagnostopoulos I, et al. Aberrantly expressed c-Jun and JunB are a hallmark of Hodgkin lymphoma cells, stimulate proliferation and synergize with NF-kappa B. EMBO J 2002;21:4104-4113.

5 Watanabe M, Ogawa Y, Ito K, et al. AP-1 mediated relief of repressive activity of the CD30 promoter microsatellite in Hodgkin and Reed-Sternberg cells. Am J Pathol 2003;163:633-641.

6 Mao X, Orchard G, Lillington DM, et al. Amplification and overexpression of JUNB is associated with primary cutaneous T-cell lymphomas. Blood 2003;101:15131519.

7 Rassidakis GZ, Georgakis GV, Oyarzo M, et al. Lack of c-kit (CD117) in CD30+ lymphomas and lymphomatoid papulosis. Mod Pathol 2004;17:946-953.

8 Cho-Vega JH, Rassidakis GZ, Amin HM, et al. Suppressor of cytokine signaling 3 expression in anaplastic large cell lymphoma. Leukemia 2004;18:1872-1878.

9 Jaffe ES, Harris NL, Stein H, Vardiman JW (eds). World Health Organization Classification of Tumours. Pathology and Genetics of Tumors of Haematopoietic and Lymphoid Tissues. World Health Organization Classification of Tumours. IARC Press: Lyon, France, 2001.

10 Rassidakis GZ, Jones D, Thomaides A, et al. Apoptotic rate in peripheral T-cell lymphomas: a study using a tissue microarray with validation on full tissue sections. Am J Clin Pathol 2002;118:328-334.

11 Rassidakis GZ, Sarris AH, Herling M, et al. Differential expression of BCL-2 family proteins in ALK-positive and ALK-negative anaplastic large cell lymphoma of T/null-cell lineage. Am J Pathol 2001;159:527-535.

12 Eferl R, Wagner EF. AP-1: a double-edged sword in tumorigenesis. Nat Rev Cancer 2003;3:859-868.

13 Nateri AS, Riera-Sans L, Da Costa C, et al. The ubiquitin ligase SCFFbw7 antagonizes apoptotic JNK signaling. Science 2004;303:1374-1378.

14 Gao M, Labuda $\mathrm{T}$, Xia $\mathrm{Y}$, et al. Jun turnover is controlled through JNK-dependent phosphorylation of the E3 ligase Itch. Science 2004;306:271-275.

15 Passegue E, Wagner EF. JunB suppresses cell proliferation by transcriptional activation of p16(INK4a) expression. EMBO J 2000;19:2969-2979.

16 Yang MY, Liu TC, Chang JG, et al. JunB gene expression is inactivated by methylation in chronic myeloid leukemia. Blood 2003;101:3205-3211.

17 Passegue E, Wagner EF, Weissman IL. JunB deficiency leads to a myeloproliferative disorder arising from hematopoietic stem cells. Cell 2004;119:431-443.

18 Szremska AP, Kenner L, Weisz E, et al. JunB inhibits proliferation and transformation in B-lymphoid cells. Blood 2003;102:4159-4165. 